This item was submitted to Loughborough's Research Repository by the author.

Items in Figshare are protected by copyright, with all rights reserved, unless otherwise indicated.

\title{
Effects of priming and pacing strategy on oxygen-uptake kinetics and cycling performance
}

\section{PLEASE CITE THE PUBLISHED VERSION}

http://dx.doi.org/10.1123/ijspp.2015-0292

\section{PUBLISHER}

(c) Human Kinetics Publishing as accepted for publication

\section{VERSION}

AM (Accepted Manuscript)

\section{PUBLISHER STATEMENT}

This work is made available according to the conditions of the Creative Commons Attribution-NonCommercialNoDerivatives 4.0 International (CC BY-NC-ND 4.0) licence. Full details of this licence are available at: https://creativecommons.org/licenses/by-nc-nd/4.0/

\section{LICENCE}

CC BY-NC-ND 4.0

\section{REPOSITORY RECORD}

Bailey, Stephen, Anni Vanhatalo, Matthew Black, Fred J. DiMenna, and Andrew M. Jones. 2019. "Effects of Priming and Pacing Strategy on Oxygen-uptake Kinetics and Cycling Performance". figshare. https://hdl.handle.net/2134/24432. 


\section{Effects of priming and pacing strategy on $\mathrm{VO}_{2}$}

\section{2 kinetics and cycling performance}

3

4 Original Investigation

5

6 Stephen J. Bailey ${ }^{\mathbf{1}}$, Anni Vanhatalo ${ }^{\mathbf{1}}$, Matthew I. Black ${ }^{\mathbf{1}}$,

7 Fred J. DiMenna ${ }^{2} \&$ Andrew M. Jones ${ }^{1}$

8

9

$10{ }^{1}$ Sport and Health Sciences, College of Life and Environmental 11 Sciences, St. Luke's Campus, University of Exeter, Heavitree Road, 12 Exeter, Devon, England, UK; ${ }^{2}$ Teachers College, Department of 13 Biobehavioral Sciences, Columbia University, New York, New York, 14 USA.

17 Correspondence:

18 Stephen J Bailey, Ph.D.

19 E-mail: S.J.Bailey@exeter.ac.uk

20 Tel: 01392722882

21 Fax: 01392264726

22

23 Running Title: Pacing, priming, $\dot{V} \mathrm{O}_{2}$ kinetics, performance

24 Abstract word count: 249 words 
ABSTRACT

Purpose: To assess whether combining prior 'priming' exercise with an all-out pacing strategy was more effective at improving $\mathrm{O}_{2}$ uptake $\left(\dot{V}_{\mathrm{O}_{2}}\right)$ kinetics and cycling performance than either intervention administered independently. Methods: Nine males completed targetwork cycling performance trials using a self-paced or all-out pacing strategy with or without prior severe-intensity $(70 \% \Delta)$ priming exercise. Breath-by-breath pulmonary $\dot{V}_{\mathrm{O}_{2}}$ and cycling power output were measured during all trials. Results: Compared to the self-pacedunprimed control trial $(22 \pm 5 \mathrm{~s})$, the $\dot{V}_{\mathrm{O}_{2}}$ mean response time (MRT) was shorter $\left(\dot{V}_{\mathrm{O}_{2}}\right.$ kinetics was faster) with all-out pacing $(17 \pm 4 \mathrm{~s})$ and priming $(17 \pm 3 \mathrm{~s})$, with the lowest $\mathrm{V}_{2}$ MRT observed when all-out pacing and priming were combined $(15 \pm 4 \mathrm{~s}) \quad(P<0.05)$. However, total $\mathrm{O}_{2}$ consumed and end-exercise $\dot{V}_{\mathrm{O}_{2}}$ were only higher than the control condition in the primed trials $(P<0.05)$. Similarly, cycling performance was improved compared to control $(98 \pm 11 \mathrm{~s})$ in the the self-paced-primed $(93 \pm 8 \mathrm{~s})$ and all-out-primed (92 \pm 8 s) trials $(P<0.05)$, but not the all-out-unprimed trial $(97 \pm 5 \mathrm{~s} ; P>0.05)$. Conclusions: These findings suggest that combining an all-out start with severe-intensity priming exercise additively improves the $\dot{V}_{\mathrm{O}_{2}}$ MRT, but not total $\mathrm{O}_{2}$ consumption and cycling performance since these were improved by a similar magnitude in both primed trials relative to the selfpaced-unprimed control condition. Therefore, these results support the use of priming exercise as a pre-competition intervention to improve oxidative metabolism and performance during short-duration high-intensity cycling exercise, independent of the pacing strategy adopted.

Key Words: Pulmonary $\dot{V}_{2}$, warm-up exercise, fast/all-out start, near-infrared spectroscopy, exercise performance 


\section{INTRODUCTION}

68 Cycling performance is a function of the power required to overcome resistive forces (e.g., air and rolling resistance) and power generation from the contracting skeletal muscles. ${ }^{1-2}$ The potential of the skeletal muscles to maintain a high power output is influenced by the energy contribution from aerobic and anaerobic metabolism. ${ }^{3-4}$ Whilst oxidative ATP turnover increases exponentially following the onset of exercise, muscle ATP demand increases immediately, which mandates an important energy contribution from anaerobic metabolism in the initial stages of exercise. ${ }^{5}$ At a given rate of ATP turnover, speeding the rate at which pulmonary oxygen uptake $\left(\dot{V} \mathrm{O}_{2}\right)$ increases over the initial stages of exercise would be expected to attenuate the reliance on the finite anaerobic energy reserves and blunt the accumulation of metabolites linked to the process of muscle fatigue. ${ }^{5}$ Therefore, interventions that enhance pulmonary $\dot{V} \mathrm{O}_{2}$ kinetics would be hypothesised to increase mean skeletal muscle power output during short-duration high-intensity exercise, permitting a higher cycling speed and a faster race completion time. ${ }^{6}$

81

Pulmonary $\check{V}_{\mathrm{O}_{2}}$ rises with more rapid overall response kinetics after prior 'priming' exercise compared to control ${ }^{7-9}$ and also when exercise is initiated with a fast-start or all-out strategy compared to even-start and slow-start strategies. ${ }^{10-15}$ Moreover, performing priming exercise prior to, ${ }^{9}$ or adopting fast-start or all-out pacing strategies during, ${ }^{11,15}$ very high work rates where fatigue ensues before the peak $\dot{V}_{\mathrm{O}_{2}}\left(\dot{V}_{\mathrm{O}_{2} \text { peak }}\right)$ can be attained (i.e., extreme-intensity exercise), ${ }^{16}$ increases the percentage of the $\dot{V}_{\mathrm{O}_{2} \text { peak }}$ that can be achieved. In addition to improving aspects of $\hat{V}_{2}$ kinetics, priming exercise and fast-start or all-out pacing strategies have been shown to improve exercise tolerance ${ }^{7,9,14}$ and performance. ${ }^{8,10-12,15,17-22}$ Since the use of prior 'warm up' exercise and fast-start strategies are recommended as interventions to enhance $\dot{V}_{\mathrm{O}_{2}}$ kinetics and athletic performance, ${ }^{23}$ understanding if and how priming exercise and different pacing strategies interact might help inform best practice for optimizing exercise performance.

The purpose of this study was to investigate whether combining prior severe-intensity priming exercise with an all-out pacing strategy would have an additive effect on the improvements in performance and $\hat{V}_{\mathrm{O}_{2}}$ kinetics that have been reported when either of these interventions is applied independently. We hypothesised that, compared to a self-pacedunprimed control condition, time-trial performance, $\dot{V} \mathrm{O}_{2}$ kinetics, total $\mathrm{O}_{2}$ consumption and the percentage of $\dot{V}_{\mathrm{O}_{2 p e a k}}$ attained would be improved by a similar extent in a self-pacedprimed trial and an all-out-unprimed trial, but that the greatest improvement in these parameters would occur when severe-intensity priming exercise and an all-out pacing strategy were combined.

104

105

106

\section{METHODS}

\section{Subjects}

107 Nine competitive male athletes (mean \pm SD: age $20 \pm 1 \mathrm{yr}$, stature $1.82 \pm 0.06 \mathrm{~m}$, body mass

$10877 \pm 8 \mathrm{~kg}$ ) volunteered to participate in this study. The study was approved by the University

109 of Exeter Research Ethics Committee and all subjects were required to give their written

110 informed consent prior to the commencement of the study. Subjects were instructed to arrive

111 at the laboratory in a rested and fully hydrated state, at least 3-h postprandial, and to avoid strenuous exercise in the 24 -h preceding each testing session.

\section{Experimental Overview}

116 period with the seven visits being separated by at least 48-h. Following the completion of 
117 preliminary exercise tests, all subjects completed four exercise performance trials (visits 4-7)

118 during which pulmonary $\dot{V} \mathrm{O}_{2}$, blood [lactate], muscle (de)oxygenation and exercise

119 performance were assessed. To determine a potential interaction between pacing strategy and priming exercise on performance and the physiological responses during exercise, we employed a paradigm comprising two different pacing strategies (self-paced and all-out) that were completed with and without priming exercise.

\section{Incremental Test}

On the first laboratory visit, subjects completed a ramp incremental cycling test for determination of the $\dot{V}_{\mathrm{O}_{2 p e a k}}$, gas exchange threshold (GET) and the work rate that would require $70 \% \Delta$ (GET plus $70 \%$ of the difference between the work rate at the GET and $\dot{V}$ $\mathrm{O}_{2 \text { peak }}$ ) as described previously. ${ }^{7}$

\section{Familiarization Trials}

During the first familiarization trial (visit 2), subjects were familiarised to the 'standing' start and were required to complete three $40 \mathrm{~kJ}$ trials lasting approximately 100 -s. The resistance on the pedals during the trials was set for each individual using the linear mode of the Lode ergometer so that the subject would attain the power output associated with $70 \% \Delta$ on reaching their preferred cadence (linear factor $=$ power/preferred cadence ${ }^{2}$ ). Subjects were provided with a 5-s countdown prior to the commencement of all cycling trials. In addition to a warm up, the first trial was used to familiarize subjects to the fixed resistance that would be imposed in all subsequent trials. In this first trial, subjects were instructed to complete the $40 \mathrm{~kJ}$ warm up by cycling at a submaximal cadence of 70-90 rpm. Following a 10-min passive recovery period, subjects repeated the $40 \mathrm{~kJ}$ trial but, on this occasion, they were instructed to complete the $40 \mathrm{~kJ}$ in the fastest time possible using a self-selected pacing strategy. Following a further 25-30-min passive recovery, subjects completed a third $40 \mathrm{~kJ}$ trial using an 'all-out' pacing strategy. The power output was continuously recorded at 5-Hz during these trials and averaged into 1-s bins for subsequent analysis. To estimate the work required for a completion time of 100-s for each individual subject, the mean power output during the self-paced trial was multiplied by 100 . This individualized work target was set during all subsequent experimental trials in an attempt to yield a completion time reflective of a $1000-\mathrm{m}$ track cycling performance for a trained but sub-elite cyclist. ${ }^{24}$

During the second familiarization trial, subjects were familiarized to the priming exercise protocol and completed two additional trials at their individualized work target. The priming exercise protocol comprised 4-min of baseline cycling at $20 \mathrm{~W}$ before an abrupt transition to the severe-intensity target work rate $(70 \% \Delta)$. The severe-intensity priming bout was 5 -min in duration. Following a 17-min passive recovery, subjects remounted the cycle ergometer and rested for an additional 3-min. This priming regime was selected since it has been shown to be particularly effective at improving performance during subsequent high-intensity cycling exercise. $^{7} \quad$ Subjects then completed their individualized work target as quickly as possible using a self-paced pacing strategy. Following 25-30-min passive recovery, subjects completed a third performance trial using an 'all-out' pacing strategy. Therefore, all subjects completed 5 repetitions of the performance trial and one repetition to the priming bout prior to the experimental testing.

\section{Experimental Trials}

In a randomized order, subjects completed self-paced and all-out trials with and without severe-intensity priming exercise over four separate experimental trials. Subjects were instructed to complete each trial as quickly as possible. Each trial was preceded by 3 -min of 
resting baseline on the cycle ergometer. Ten seconds prior to the commencement of each trial, subjects were instructed to adjust the crank angle to their preferred starting position, which was established in the familiarization trials and replicated in all experimental trials, and to assume a standing position on the cycle ergometer. Subjects were then provided with a 5-s countdown to indicate when the trial would commence. For the initial 10-s of the trial, subjects were required to cycle in the upright position before being instructed to assume a seated position for the remainder of the trial. Subjects were made aware of their work target prior to each trial and the work target and accrued work during the trial was displayed on a computer screen placed directly in front of the subject. Strong verbal encouragement was provided during all trials, but subjects were not aware of the elapsed time during the trials.

\section{Measurements}

All cycle tests were performed on an electrically-braked cycle ergometer (Lode Excalibur Sport, Groningen, the Netherlands). During all tests, pulmonary gas exchange and ventilation were measured breath-by-breath using an online gas analyzer (Jaeger Oxycon Pro, Hoechberg, Germany), muscle oxygenation variables (deoxygenated hemoglobin concentration $[\mathrm{HHb}]$, oxygenated hemoglobin concentration $\left[\mathrm{O}_{2} \mathrm{Hb}\right]$, total hemoglobin concentration $\left[\mathrm{Hb}_{\text {tot }}\right]$ and tissue oxygenation index (TOI)) were measured using near-infrared spectroscopy (model NIRO 300, Hamamatsu Photonics KK, Hiugashi-ku, Japan) and a blood sample was collected from a fingertip into a capillary tube 30 -s prior to the commencement of the trial and immediately following the trial for blood [lactate] determination (YSI 1500, Yellow Springs Instruments, Yellow Springs, OH, United States), as described previously. ${ }^{11}$

\section{Data Analysis Procedures}

Prior to analysis the breath-by-breath $\dot{V}_{2}$ data from each test were treated as described previously. ${ }^{11}$ A single-exponential model without time delay, with the fitting window commencing at $t=0 \mathrm{~s}$ (equivalent to the mean response time, MRT) was used to characterize the kinetics of the overall $\dot{V}_{\mathrm{O}_{2}}$ response during the trials as described in the following equation:

$$
\dot{V} \mathrm{O}_{2}(\mathrm{t})=\dot{V} \mathrm{O}_{2} \text { baseline }+\mathrm{A}\left(1-\mathrm{e}^{-\mathrm{t} / \mathrm{MRT})}\right)
$$

where $\dot{V}_{\mathrm{O}_{2}}(t)$ represents the absolute $\dot{V}_{\mathrm{O}_{2}}$ at a given time $t ; \dot{V} \mathrm{O}_{2}$ baseline represents the mean $\dot{V}_{\mathrm{O}_{2}}$ measured over the final 90-s of baseline; and A and MRT represent the amplitude and MRT, respectively, describing the overall increase in $\dot{V}_{\mathrm{O}_{2}}$ above baseline. An iterative process was used to minimize the sum of the squared errors between the fitted function and the observed values. We quantified the $\dot{V}_{\mathrm{O}_{2}}$ MRT with the fitting window constrained to both completion time (end-exercise) and at the minimum completion time for each subject across the four experimental trials $\left(\mathrm{T}_{\text {min }}\right)$. The absolute $\dot{V}_{2}$ at, and the total $\mathrm{O}_{2}$ consumed up to, 60-s $( \pm 5-\mathrm{s})$, end-exercise (average over the final 10-s) and $\mathrm{T}_{\min }$ (average over the final 10-s) were also calculated. We also divided the total $\mathrm{O}_{2}$ consumed up to 60 -s by the work accumulated over the corresponding time frame to provide an indication of the oxidative energy provision relative to external power output.

The $[\mathrm{HHb}]$ kinetics during the exercise bouts was determined by fitting a mono-exponential model with a time delay (TD) from the first data point which was 1 SD above the baseline mean. The $[\mathrm{HHb}] \mathrm{TD}$ and $\tau$ values were summed, to provide information on the overall $[\mathrm{HHb}]$ response kinetics. We quantified the $[\mathrm{HHb}]$ kinetics during the trials using three different fitting procedures: 1) the fitting window was constrained to the point at which mono-exponentiality became distorted, consequent to a gradual fall in $[\mathrm{HHb}]$, as determined 
217 by visual inspection of the residual plots data (peak fit); 2) the fitting window was

218 constrained to $\mathrm{T}_{\min }\left(\mathrm{T}_{\min }\right.$ fit); and 3 ) the $\mathrm{HHb}$ data were fit to end-exercise (end-exercise fit).

219 The $[\mathrm{HHb}],\left[\mathrm{O}_{2} \mathrm{Hb}\right],\left[\mathrm{Hb}_{\text {tot }}\right]$ and TOI values at baseline (average over the 90 -s preceding the

220 onset of the trial), 20-s ( \pm 5 -s), 60-s ( \pm 5 -s) and end-exercise (average over the final 10-s)

221 were also calculated.

Performance during the fixed work trial was determined by the time required to complete the designated work target. Peak power output during the trials was taken as the highest 1-s power output during the trial and end-exercise power output was taken as the average power output over the final 10-s of the trial.

\section{Statistical Analysis}

A two-way (pacing x priming) repeated-measures ANOVA was employed to determine the effects of pacing strategy and priming exercise on the relevant physiological and performance variables. Where the analysis revealed a significant difference, individual paired $t$-tests were employed with a Fisher's LSD to determine the origin of such effects. All data are presented as mean $\pm \mathrm{SD}$. Statistical significance was accepted when $P<0.05$.

\section{RESULTS}

During the ramp incremental test, subjects attained a peak work rate of $370 \pm 45 \mathrm{~W}$ and a $\dot{V}$ $\mathrm{O}_{2 \text { peak }}$ of $4.18 \pm 0.56 \mathrm{~L} \cdot \mathrm{min}^{-1}$. The work target for the performance trials was $41.3 \pm 4.8 \mathrm{~kJ}$ and the work rate applied during the severe-intensity priming bout was $273 \pm 37 \mathrm{~W}$.

\section{Blood [lactate]}

Baseline blood [lactate] was greater in the primed trials $(P<0.001$; Table 1). End-exercise blood [lactate] was higher in the self-paced-primed and all-out-primed trials compared to the self-paced-unprimed control trial $(P<0.05)$, but not the all-out-unprimed trial $(P>0.05$; Table 1).

\section{Near-infrared Spectroscopy}

Baseline muscle $\left[\mathrm{O}_{2} \mathrm{Hb}\right],\left[\mathrm{Hb}_{\text {tot }}\right]$ and TOI were higher in the primed trials $(\mathrm{P}<0.05$; Table 2$)$. Muscle $\left[\mathrm{O}_{2} \mathrm{Hb}\right]$ and $\left[\mathrm{Hb}_{\text {tot }}\right]$ were greater during exercise in the primed trials, whereas TOI was higher 20-s into exercise in the primed trials compared to the all-out-unprimed condition $(\mathrm{P}<0.05$; Table 2). Muscle [HHb] $\tau+\mathrm{TD}$ was shorter in both primed trials compared to the self-paced-unprimed control $(P<0.05$; Figure 1; Table 2).

\section{Vo $o_{2}$ Kinetics}

Compared to the self-paced-unprimed control, the $\dot{V}_{\mathrm{O}_{2}}$ MRT was shorter in all other experimental conditions $(P<0.05)$. Moreover, the $V_{\mathrm{O}_{2}}$ MRT was shorter in the all-out-primed compared to the all-out-unprimed and self-paced-primed conditions $(P<0.05$; Table 3; Figure 2). The total $\mathrm{O}_{2}$ consumed and the total $\mathrm{O}_{2}$ consumed relative to work done over the first 60$\mathrm{s}$ of exercise were greater in the self-paced-primed and all-out primed trials compared to their respective unprimed conditions $(P<0.01$; Table 3$)$. In the unprimed trials the end-exercise $V$ $\mathrm{O}_{2}$ was lower than the ramp test $\dot{V} \mathrm{O}_{2 \text { peak }}$ and the end-exercise $\dot{V}_{\mathrm{O}_{2}}$ during the primed trials $(P<0.05)$, whereas the end-exercise $\dot{V}_{\mathrm{O}_{2}}$ during the primed trials was not different from the $\dot{V}$ O2peak $(P>0.05$; Table 3$)$.

\section{Cycling Performance}

The peak power output and total work done over the first 60 -s were higher in the all-out trials $(P<0.05)$, whereas end-exercise power output was higher with priming $(P<0.05$; Figure 3$)$. 
Trial completion time was faster than control (98 \pm 11 -s) in the self-paced (93 \pm 8 -s) and allout $(92 \pm 8$-s; both $P<0.05)$ primed trials, but not with all-out pacing alone $(97 \pm 5$-s; $P>0.05$; Figure 4). Completion time was also shorter in the all-out trial after priming compared to the

\section{DISCUSSION}

The principal original findings from this study are that muscle (de)oxygenation, pulmonary $\dot{V}$ $\mathrm{O}_{2}$ and performance were similar during short-duration high-intensity cycling exercise initiated with a self-paced or all-out pacing strategy in the unprimed state, but that these variables were enhanced by a similar magnitude when either of these pacing strategies was preceded by a bout of priming exercise. These findings might have important implications for performance enhancement in short-duration high-intensity events, such as 1000-m track cycling, and suggest that priming exercise is similarly effective at improving muscle (de)oxygenation, pulmonary $\mathrm{V}_{2}$ and cycling performance irrespective of whether an all-out or self-paced pacing strategy is applied.

When all-out pacing and priming were combined, the $\dot{V}_{\mathrm{O}_{2}} \mathrm{MRT}$ (when modelled to $\mathrm{T}_{\min }$ ) was $12 \%$ smaller compared to either intervention administered independently, or $32 \%$ smaller than the control trial. The $V_{O_{2}}$ MRT was $23 \%$ smaller compared to the control trial with priming or all-out pacing alone. Faster overall $\dot{V}_{2}$ kinetics have been reported in previous studies following priming exercise ${ }^{7-9,25}$ and when fast start strategies are employed. ${ }^{11,13-15,25}$ In contrast to the findings of this study, a recent study observed no additive effect of combining heavy-intensity priming and a fast-start strategy on the $\tilde{V}_{2} \mathrm{MRT}^{25}$ These conflicting findings might be linked to between-study differences in priming intensity and pacing strategies, and the potential for more rapid $\hat{V}_{2}$ kinetics with the severe-intensity priming ${ }^{7}$ and all-out pacing strategy ${ }^{15}$ used in the current study, relative to the heavy-intensity priming and fast-start strategy imposed by Caritá et al. ${ }^{25}$ Nonetheless, despite an additive improvement in the $\dot{V}_{\mathrm{O}_{2}}$ MRT, the total $\mathrm{O}_{2}$ consumed up to $\mathrm{T}_{\min }$ and the $\dot{V}_{\mathrm{O}_{2}}$ attained at endexercise were higher in both primed trials, but were not different between the two primed trials or between the two unprimed trials. Indeed, subjects were able to attain their $\dot{V}_{\text {ozpeak }}$ (i.e., as measured on the initial ramp test) during the short-duration cycling bouts after priming regardless of pacing strategy employed whereas without priming, they were not. This is consistent with reports that priming exercise permits the attainment of $\dot{V}_{\mathrm{O}_{\text {peak }}}$ during extreme-intensity exercise where $\dot{V}_{\text {Oppeak }}$ is not attained in the unprimed condition. ${ }^{9}$ Therefore the attainment of $\bar{V}_{\mathrm{O}_{2} \text { peak }}$ with priming permitted a greater total $\mathrm{O}_{2}$ consumption, whereas the faster $\mathrm{V}_{2}$ kinetics with an all-out start was not sufficient to increase total $\mathrm{O}_{2}$ consumption as the percentage of $\dot{V}_{2} o_{2 e a k}$ attained was not significantly altered.

Muscle blood flow at rest and during the initial stages of exercise has been shown to increase after completing intense priming exercise. ${ }^{26-27}$ Our findings of a greater muscle [Hbtot], $\left[\mathrm{O}_{2} \mathrm{Hb}\right]$ and TOI with priming are compatible with previous reports of improved muscle perfusion and $\mathrm{O}_{2}$ availability after priming exercise. ${ }^{726-27}$ Therefore, enhanced muscle perfusion and $\mathrm{O}_{2}$ availability in the primed trials might have contributed towards the more rapid $\dot{V}_{\mathrm{O}_{2}}$ kinetics, greater total $\mathrm{O}_{2}$ consumption and attainment of a greater percentage of $\dot{V}$ O2peak compared to the unprimed conditions. 3,28 However, in addition to greater muscle $\mathrm{O}_{2}$ delivery, enhanced muscle $\mathrm{O}_{2}$ extraction ${ }^{26-27}$ and faster muscle $[\mathrm{HHb}]$ kinetics ${ }^{7}$ have also been previously reported following priming exercise. In line with these findings, muscle $[\mathrm{HHb} \tau \tau$ $+\mathrm{TD}$ was shorter with priming in this study, suggestive of enhanced fractional $\mathrm{O}_{2}$ extraction contributing to faster $\tilde{V}_{\mathrm{O}_{2}}$ kinetics following priming. ${ }^{29}$ Therefore, faster $\dot{V} \mathrm{O}_{2}$ kinetics, attainment of a greater percentage of $\dot{V}_{2}$ peak and greater $\mathrm{O}_{2}$ consumption after priming 
317 exercise in this study are likely to have arisen as a result of a positive interaction between

318 improvements in muscle $\mathrm{O}_{2}$ supply and $\mathrm{O}_{2}$ extraction.

319

320

321

322

323

324

325

326

327

328

329

330

331

332

333

334

335

336

337

338

339

340

341

342

343

344

345

346

347

348

349

350

351

352

353

354

355

356

357

358

359

360

361

362

363

364

365

366

Although total $\mathrm{O}_{2}$ consumption over the initial 60 -s of exercise was greater with priming, changes in total $\mathrm{O}_{2}$ consumption between the experimental conditions were not proportional to alterations in power output in all conditions. Commencing exercise at a higher power output, as observed when all-out pacing strategies are employed, would be expected to promote more rapid increases in aerobic, anaerobic and total ATP turnover rates. ${ }^{30}$ Therefore, while $\mathrm{V}_{2}$ increased more rapidly in the all-out trials relative to the self-pacedunprimed trial, this potential for an increased aerobic energy yield in the all-out conditions was accompanied by a greater total work done over the initial stages of exercise. Accordingly, the $\mathrm{O}_{2}$ consumed per unit work, and presumably the proportional aerobic energy contribution, was not significantly different from the self-paced-unprimed trial in either all-out trial over the first 60-s of exercise. However, since priming exercise does not increase the total ATP turnover rate in a subsequent bout of exercise at the same absolute work rate ${ }^{26-27}$ and since the pattern of work rate distribution over the first 60-s was similar for primed and unprimed conditions when the same pacing strategy was employed, the total ATP turnover rate and its temporal fluctuation might be expected to be similar between the two self-paced trials, and the two all-out trials. The $\mathrm{O}_{2}$ consumed per unit work over the first $60-\mathrm{s}$ was higher after priming ( $\sim 9 \%$ and $\sim 7 \%$ for self-paced-primed compared to self-pacedunprimed and all-out-primed compared to all-out-unprimed, respectively). This is suggestive of a greater proportional aerobic energy contribution in the self-paced-primed and all-outprimed trials relative to their respective unprimed conditions. Consistent with this interpretation, intense priming exercise has been shown to increase aerobic ATP turnover and lower anaerobic ATP turnover, without altering the total ATP turnover, during the initial stages of a subsequent bout of intense constant work rate exercise. ${ }^{26-27}$

Cycling performance was not significantly impacted by the pacing strategy employed in this study in either the primed or unprimed trials. While the $\dot{V}_{\mathrm{O}_{2}}$ MRT was lower in the all-outunprimed trial compared to the self-paced-unprimed trial, $\dot{V}_{\mathrm{O} 2 \text { peak }}$ was not attained in either of these trials and $\mathrm{O}_{2}$ consumed, and $\mathrm{O}_{2}$ consumed relative to work done over the first 60-s, were similar between trials. We have previously shown that fast-start ${ }^{11}$ and all-out ${ }^{15}$ pacing strategies are ergogenic during short-duration high-intensity exercise when $\dot{V}_{\mathrm{O}_{2}}$ kinetics is faster and the percentage of $\dot{V}_{\mathrm{O}_{2} \text { peak }}$ attained is greater, but not necessarily when $\mathrm{V}_{\mathrm{O}_{2}}$ kinetics is faster without changes in the percentage of $\dot{V}_{\mathrm{O}}$ peak attained or total $\mathrm{O}_{2}$ consumed. On the other hand, the total $\mathrm{O}_{2}$ consumed and $\mathrm{O}_{2}$ consumption relative to work done over the first 60 -s were higher, the percentage of $\bar{V}_{\mathrm{O}_{2} \text { peak }}$ attained was increased and exercise performance was improved with priming when the same pacing strategy was employed. This finding is consistent with previous reports that priming exercise is ergogenic ${ }^{7-9,19-21,25}$, particularly when baseline blood [lactate] is elevated to $3-4 \mathrm{mM}^{8-9,11}$ and suggests that priming might improve short-duration high-intensity exercise performance by increasing the absolute aerobic energy contribution to total energy turnover. However, since the exercise performance trials in this study were conducted in competitive, but not highly trained, athletes in an exercise physiology laboratory, further research is required to assess the effects of pacing and prior exercise strategies on cycling performance in well-trained cyclists in the velodrome.

In conclusion, while $\dot{V}_{\mathrm{O}_{2}}$ kinetics was faster when an all-out pacing strategy was employed, there were no changes in muscle (de)oxygenation, total $\mathrm{O}_{2}$ consumption, the percentage of $\dot{V}$ $\mathrm{O}_{2 \text { peak }}$ attained and cycling performance between these experimental conditions. However, pulmonary $\tilde{V}_{\mathrm{O}_{2}}$ and muscle (de)oxygenation kinetics were speeded, total $\mathrm{O}_{2}$ consumption and 
the percentage of $\dot{V}_{\mathrm{O}_{2 p e a k}}$ attained were increased, and cycling performance was improved in the self-paced-primed and all-out-primed trials compared to their respective unprimed conditions. Therefore, while combining priming with an all-out start evoked additive improvements in $\mathrm{VO}_{2}$ kinetics, a similar magnitude of improvement in muscle (de)oxygenation variables, total $\mathrm{O}_{2}$ consumption and short-duration high-intensity cycling performance was observed with priming regardless of the pacing strategy adopted. These findings support the use of prior high-intensity priming exercise as a pre-competition intervention to increase oxidative energy contribution and improve performance in shortduration high-intensity events such as $1000-\mathrm{m}$ track cycling.

Acknowledgement: This research was not supported by external funding. The authors are grateful to Joshua Bartlett, Myles Blenkinsop and Ian White for assistance during data collection. 
418 1. Craig NP, Norton KI. Characteristics of track cycling. Sports Med. 2001;31:457-468.

2. Jeukendrup AE, Craig NP, Hawley JA. The bioenergetics of World Class Cycling. J Sci Med Sport. 2000;3:414-433.

422

3. Grassi B, Hogan MC, Kelley KM, Aschenbach WG, Hamann JJ, Evans RK, Patillo RE, Gladden LB. Role of convective $\mathrm{O}_{2}$ delivery in determining $\dot{V}_{2}$ on-kinetics in canine muscle contracting at peak $\dot{V}_{2}$. J Appl Physiol. 2000;89:1293-1301.

4. Sirikul B, Hunter GR, Larson-Meyer DE, Desmond R, Newcomer BR. Relationship between metabolic function and skeletal muscle fatigue during a $90 \mathrm{~s}$ maximal isometric contraction. Appl Physiol Nutr Metab. 2007;32:394-399.

5. Poole DC, Jones AM. Oxygen uptake kinetics. Compr Physiol. 2012;2:933-996.

6. Burnley M, Jones AM. Oxygen uptake kinetics as a determinant of sports performance. Eur J Sports Sci. 2007;7:63-79.

7. Bailey SJ, Vanhatalo A, Wilkerson DP, Dimenna FJ, Jones AM. Optimizing the "priming" effect: influence of prior exercise intensity and recovery duration on $\mathrm{O} 2$ uptake kinetics and severe-intensity exercise tolerance. J Appl Physiol. 2009;107:1743-1756.

8. Burnley M, Doust JH, Jones AM. Effects of prior warm-up regime on severe-intensity cycling performance. Med Sci Sports Exerc. 2005;37:838-45.

9. Jones AM, Wilkerson DP, Burnley M, Koppo K. Prior heavy exercise enhances performance during subsequent perimaximal exercise. Med Sci Sports Exerc. 2003;35:20852092.

10. Aisbett B, Lerossignol P, McConell GK, Abbiss CR, Snow R. Effects of starting strategy on 5-min cycling time-trial performance. J Sports Sci. 2009b;27:1201-1209.

11. Bailey SJ, Vanhatalo A, DiMenna FJ, Wilkerson DP, Jones AM. Fast-start strategy improves $\dot{V}_{\mathrm{O}_{2}}$ kinetics and high-intensity exercise performance. Med Sci Sports Exerc. 2011;43:457-467.

12. Bishop D, Bonetti $\mathrm{D}$, Dawson $\mathrm{B}$. The influence of pacing strategy on $\dot{V}_{\mathrm{O}}$ and supramaximal kayak performance. Med Sci Sports Exerc. 2002;34:1041-1047.

13. Hettinga $\mathrm{FJ}$, de Koning JJ, Foster C. $\dot{V} \mathrm{O}_{2}$ response in supramaximal cycling time trial exercise of 750 to 4000 m. Med Sci Sports Exerc. 2009;41:230-236.

14. Jones AM, Wilkerson DP, Vanhatalo A, Burnley M. Influence of pacing strategy on $\mathrm{O}_{2}$ uptake and exercise tolerance. Scand J Med Sci Sports. 2008;18:615-626.

15. Wood MA, Bailey SJ, Jones AM. Influence of all-out start duration on pulmonary oxygen uptake kinetics and high-intensity exercise performance. J Strength Cond Res. 2014;28:21872194. 
16. Hill DW, Poole DC, Smith JC. The relationship between power and the time to achieve $\dot{V}$ o2. Med Sci Sports Exerc. 2002;34:709-714.

17. Aisbett B, Lerossignol P, McConell GK, Abbiss CR, Snow R. Influence of all-out and fast start on 5-min cycling time trial performance. Med Sci Sports Exerc. 2009a;41:19651971.

18. de Koning JJ, Bobbert MF, Foster C. Determination of optimal pacing strategy in track cycling with an energy flow model. J Sci Med Sport. 1999;2:266-277.

19. Hajoglou A, Foster C, De Koning JJ, Lucia A, Kernozek TW, Porcari JP. Effect of warm-

20. Ingham SA, Fudge BW, Pringle JS, Jones AM. Improvement of 800-m running performance with prior high-intensity exercise. Int J Sports Physiol Perform. 2013;8:77-83.

21. Palmer CD, Jones AM, Kennedy GJ, Cotter JD. Effects of prior heavy exercise on energy supply and 4000-m cycling performance. Med Sci Sports Exerc. 2009;41:221-229.

22. van Ingen Schenau GJ, de Koning JJ, de Groot G. The distribution of anaerobic energy in 1000 and 4000 meter cycling bouts. Int J Sports Med. 1992;13:447-451.

23. Jones AM, Burnley M. Oxygen uptake kinetics: an underappreciated determinant of exercise performance. Int J Sports Physiol Perform. 2009;4:524-532.

24. Foster C, deKoning JJ, Hettinga F, Lampen J, Dodge C, Bobbert M, Porcari JP. Effect of competitive distance on energy expenditure during simulated competition. Int J Sports Med. 2004;25:198-204.

25. Caritá RA, Greco CC, Denadai BS. The positive effects of priming exercise on oxygen uptake kinetics and high-intensity exercise performance are not magnified by a fast-start pacing strategy in trained cyclists. PLoS One. 2014;9:e95202.

26. Bangsbo J, Krustrup P, González-Alonso J, Saltin B. ATP production and efficiency of human skeletal muscle during intense exercise: effect of previous exercise. Am J Physiol Endocrinol Metab. 2001;280:956-964.

27. Krustrup P, González-Alonso J, Quistorff B, Bangsbo J. Muscle heat production and anaerobic energy turnover during repeated intense dynamic exercise in humans. J Physiol. 2001;536:947-956.

28. Richardson RS, Grassi B, Gavin TP, Haseler LJ, Tagore K, Roca J, Wagner PD. Evidence of $\mathrm{O}_{2}$ supply-dependent $\mathrm{VO}_{2}$ max in the exercise-trained human quadriceps. $J$ Appl Physiol. 1999;86:1048-1053.

29. Koga S, Poole DC, Ferreira LF, Whipp BJ, Kondo N, Saitoh T, Ohmae E, Barstow TJ. Spatial heterogeneity of quadriceps muscle deoxygenation kinetics during cycle exercise. $J$ Appl Physiol. 2007;103:2049-2056. 
516 DP, Benjamin N, Jones AM. Dietary nitrate supplementation enhances muscle contractile 517 efficiency during knee-extensor exercise in humans. J Appl Physiol. 2010;109:135-148. 
566 Figure 1. Near-infrared spectroscopy-derived muscle deoxyhemoglobin concentration $([\mathrm{HHb}])$ responses in the self-paced unprimed (SP-UP) trial compared to the all-out unprimed (AO-UP) trial (panel A); the self-paced primed (SP-P) trial compared to the all-out primed (AO-P) trial (panel B); SP-UP compared to SP-P (panel C); and AO-UP compared to AO-P (panel D). Data are presented as group mean responses with the baseline normalized to 0 and expressed as the change $(\Delta)$ from baseline. The end-exercise muscle $[\mathrm{HHb}]$ is presented with $\mathrm{y}$-axis \pm SEM error bars and $\mathrm{x}$-axis \pm SEM error bars for completion time in the performance tests. The dashed vertical lines represent the start of the cycling performance trials. * indicates a significantly longer completion time relative to the respective comparison condition $(P<0.05)$.

Figure 2. Pulmonary oxygen uptake $\left(\dot{V}_{\mathrm{O}}\right)$ responses in the self-paced unprimed (SP-UP) trial compared to the all-out unprimed (AO-UP) trial (panel A); the self-paced primed (SP-P) trial compared to the all-out primed (AO-P) trial (panel B); SP-UP compared to SP-P (panel C); and AO-UP compared to AO-P (panel D). Data are presented as group mean responses with the end-exercise pulmonary $\dot{V}_{\mathrm{O}_{2}}$ presented with y-axis $\pm \mathrm{SEM}$ error bars and $\mathrm{x}$-axis $\pm \mathrm{SEM}$ error bars for completion time in the performance test. The dashed vertical lines represent the start of the cycling performance trials. * indicates a significantly longer completion time relative to the respective comparison condition $(P<0.05)$. \# indicates significantly higher pulmonary $\check{V}_{\mathrm{O}_{2}}$ relative to the respective comparison condition $(P<0.05)$.

Figure 3. Cycle ergometry power output responses in the self-paced unprimed (SP-UP) trial compared to the all-out unprimed (AO-UP) trial (panel A); the self-paced primed (SP-P) trial compared to the all-out primed (AO-P) trial (panel B); SP-UP compared to SP-P (panel C); and AO-UP compared to AO-P (panel D). Data are presented as group mean responses with the end-exercise power output presented with $y$-axis \pm SEM error bars and $x$-axis \pm SEM error bars for completion time in the performance tests. The dashed vertical lines represent the start of the cycling performance trial. * indicates a significantly longer completion time relative to the respective comparison condition $(P<0.05)$. \# indicates significantly higher power output relative to the respective comparison condition $(P<0.05)$.

Figure 4. Completion times during the target-work cycling trials in the self-paced unprimed (SP-UP), all-out unprimed (AO-UP), self-paced primed (SP-P) and all-out primed (AO-P) conditions. Data are presented as group mean responses with \pm SEM error bars. * indicates a faster completion time compared to SP-UP and AO-UP $(P<0.05)$. 